Z. klin. Chem. u. klin. Biochem.

8. Jg., S. $403-405$, Juli 1970

\title{
Einfluß von Sekretin und Pankreozymin auf die Lactosedigestion und die Wassersorption der Ratte)
}

\author{
Von R. BöHMER und K. ROMMEL \\ Aus der Sektion für Klinische Chemie (Doz. Dr. K. Rommel) des Zentrums für Innere Medizin und Kinderbeilkunde der \\ Universität Ulm - Medizinisch-Naturwissenschaftliche-Hocbscbule -
}

(Eingegangen am 10. März 1970)

Mit Hilfe der Perfusionstechnik wurde untersucht, ob Sekretin und Pankreozymin die Lactosedigestion beeinflussen. Nach 6 E. Sekretin i. $\nabla$. pro kg Körpergewicht zeigt sich eine statistisch nicht signifikante Vcrminderung der Lactoscspaltung gegenüber unbehandelten Tieren, nach 6 E. Pankreozymin i. v. pro kg Körpergewicht kommt es in dem Zeitraum 0-30 Min. nach Perfusionsbeginn zu einer statistisch signifikanten Verminderung der Lactasehydrolyse, während 30-60 Min. nach Perfusionsbeginn die Unterschiede nicht mehr signifikant sind. Als Ursache können eine Hemmung der Lactaseaktivität in der Mukosazelle oder eine Hemmung des Lactosetransportes in die Zelle durch Pankreozymin angenommen werden. Diese Mechanismen können entweder auf einer direkten Pankreozyminwirkung beruhen, oder auf einem sekundären Effekt nach Stimulierung des endokrinen Pankreas durch Pankreozymin.

Die Absorption der durch Lactosehydrolyse freigesetzten Galaktose wird in unseren Untersuchungen durch Sekretin und Pankreozymin nicht beeinflußt. Der Gehalt des Perfusates an freier Galaktose ist nach Sekretin- und Pankreozymingabe im Vergleich zu unbehandelten Kontrolltieren unverändert niedrig (um $1 \mathrm{mg} / 100 \mathrm{ml}$ ). Da Sekretin und Pankreozymin eine Insulinausschüttung bewirken, hätte man eine verminderte Galaktoseabsorption als Folge eines erhöhten Insulinspiegels erwarten können. Für die fehlende Beeinflussung der Galaktoseabsorption könnte die von uns verwendete geringe Laktosekonzentration im Perfusat $(100 \mathrm{mg} / 100 \mathrm{~m} /)$ verantwortlich sein, da eine Absorptionshemmung vielleicht erst bei höheren Galaktosekonzentrationen sichtbar wird.

Im Vergleich zu unbehandelten Kontrolltieren bewirken Sekretin und Pankreozymin eine verminderte Wasserverschiebung vom Darmlumen in das Gewebe, entweder durch vermehrte Wasserinsorption oder verminderte Wasserabsorption. Ein Verdünnungseffekt durch vermehrt ausgeschüttetes Pankreassekret nach Stimulierung durch Pankreozymin und Sekretin scheidet aufgrund der angewendeten Technik aus.

\section{The influence of secretin and pancreozymin on the digestion of lactose and the absorption of nater in the rat}

Using the perfusion technique, the influence of secretin and pancreozymin on the digestion of lactose was studied. After an i. $\nabla$. secretinload of 6 units per $\mathrm{kg}$ body weight there was a decrease of the lactose hydrolysis in comparison to untreated animals, but this was not statistically significant. After an i. v. pancreozymin-load of 6 units per $\mathrm{kg}$ body weight a statistically significant decrease of the lactose hydrolysis could be demonstrated during the time interval of $0-30 \mathrm{~min}$. after the beginning of the perfusion - whilst the differences during 30-60 $\mathrm{min}$. after the commencement of the perfusion were no more significant. These findings could be explained by an inhibition of the lactase activity of the mucosa cell or an inhibition of the transport of lactose into the cell by pancreozymin.

In comparison to untreated control animals secretin and pancreozymin cause a decreased water exchange from the gut lumen into the tissue, either by increased water insorption or by decreased water absorption. A dilution effect by an increase of pancreas secretion after stimulation by pancreozymin and secretin is excluded by the technique used.

Die zur Disaccharidspaltung im Dünndarm notwendigen Hydrolasen sind hauptsächlich im Bürstensaum der Mukosazellen lokalisiert. Im Darmlumen kommen nur minimale Disaccharidasenmengen vor, die wahrscheinlich aus einer Abschilferung von Mukosazellen entstanden sind (1). Die Hauptaktivität der Lactase liegt bei der Ratte im mittleren Drittel des Dünndarmes (2). Ziel dieser Arbeit war, zu untersuchen, ob die Lactosedigestion einer Beeinflussung durch die intestinalen Hormone Sekretin und Pankreozymin unterliegt.

\section{Methodik}

Die: Untersuchungen wurden an männlichen Wistar-Ratten von $200-250 \mathrm{~g}$ Körpergewicht nach 12-stdg. Nahrungskarenz (Wasser ad libitum) mit der Perfusionstechnik durchgeführt. Um Wasserverschiebungen durch Diffusion möglichst zu vermeiden, wählten wir als Basisperfusionslösung eine blutisotone Natriumsulfatlösung, welche außerdem $100 \mathrm{mg} / 100 \mathrm{ml}$ Lactose und $100 \mathrm{mg}$ / $100 \mathrm{ml}$ Kollidon (Polyvinylpyrrolidon) enthielt. Mit Hilfe des

1) Mit Unterstützung der Deutschen Forschungsgemeinschaft, Bad Godesberg. nicht absorbierbaren Kollidons wurde der Grad der Wasserverschiebung im Darmlumen untersucht. Die Tiere wurden mit $0,4 \mathrm{~m} l$ 5proz. Pentobarbital (Nembutal) i. p. narkotisiert. Die Perfusion erfolgte an einem $10 \mathrm{~cm}$ langen Darmstück $30-40 \mathrm{~cm}$ kaudal vom Pylorus mit konstanter Geschwindigkeit von $0,2 \mathrm{~m} l /$ Min. Zur Angleichung an die Körpertemperatur des Tieres leiteten wir die Perfusionslösung durch ein erwärmtes Wasserbad. Ein Austrocknen des Darmes wurde durch Zurückverlagern in die Bauchhöhle und Bedecken mit feuchten Tüchern vermieden. Nach Passieren des untersuchten Darmsegmentes ivurden 6 Proben über jeweils $10 \mathrm{Min}$. in einem Fraktionssammler getrennt gesammelt. Die Lactosebestimmung erfolgte durch enzymatische Bestimmung der freigesetzten Galaktose (Testpack Boehringer Mannheim) nach Lactosespaltung mit $\beta$-Galaktosidase. Außerdem wurde in jeder Probe die freie Galaktose vor Lactosespaltung bestimmt.

Die Kollidonbestimmung wurde in Anlehnung an die von SCHUBERT und WERNER (3) angegebene Methode durchgeführt. $0,5 \mathrm{~m} l$ Probelösung wurden mit $0,1 \mathrm{~m} / 3$ proz. Trichloressigsäure enteiweißt und zentrifugiert, anschlicßend wurden $0,5 \mathrm{~m} /$ des Uberstandes mit $2 \mathrm{~m} /$ Jod-Jodkali-Lösung und $2 \mathrm{ml}$ 10proz. $\mathrm{HCl}$ versetzt. Der ausgefällte Kollidon-Jod-Komplex wurde $10 \mathrm{Min}$. bei $3000 \mathrm{U}$./Min. abzentrifugiert, der Überstand abgegossen. Das Sediment wurde mit $5 \mathrm{~m} /$ absol. Methanol aufgenommen und gelöst. Vom Uberstand wurden $0,5 \mathrm{~m} / \mathrm{mit} 2 \mathrm{~m} /$ 
absol. Methanol verdünnt und die Extinktion am EppendorfPhotometer bei $436 \mathrm{~nm}$ gegen Methanol gemessen. An einer stets mitgeführten Eichkurve wurden die Kollidonkonzentrationen $(\mathrm{mg} / 100 \mathrm{~m} l)$ abgelesen.

Die Lactosedigestion wurde nach der Formel:

$$
\mathrm{D}=100 \cdot\left(1-\frac{\mathrm{L}_{\mathbf{P}} \cdot \mathrm{K}_{\mathbf{S}}}{\left.\mathrm{K}_{\mathbf{P}} \cdot \mathrm{L}_{\mathbf{S}}\right)}\right)
$$

berechnet.

$\mathrm{L}_{\mathrm{S}}=$ Lactosegehalt der Stammlösung $(\mathrm{mg} / 100 \mathrm{~m} l)$

$\mathrm{K}_{\mathrm{s}}=$ Kollidongehalt der Stammlösung $(\mathrm{mg} / 100 \mathrm{ml})$

$\mathrm{L}_{\mathrm{P}}=$ Lactosegehalt det Probe nach Perfusion $(\mathrm{mg} / 100 \mathrm{ml}$ )

$\mathrm{K}_{\mathrm{P}}=$ Kollidongehalt der Probe nach Perfusion $(\mathrm{mg} / 100 \mathrm{ml}$ )

\section{Ergebnisse}

Die Ergebnisse für die Lactosedigestion zeigen die Tabelle 1 und die Abbildung 1. Die Lactosespaltung ist nach Sekretin und Pankreozymin vermindert gegenüber

Tab. 1

Absorbierte Lactosemenge in Prozent vom Ausgangswert

\begin{tabular}{|c|c|c|c|c|c|c|}
\hline \multirow{2}{*}{$\begin{array}{l}\text { Perfusionsdauer } \\
\text { (Min.) }\end{array}$} & \multicolumn{2}{|c|}{ Kontrolltiere } & \multicolumn{2}{|c|}{$\begin{array}{l}\text { nach } 6 \text { E. } \\
\text { Sekretin/kg }\end{array}$} & \multicolumn{2}{|c|}{$\begin{array}{c}\text { nach } 6 \text { E. } \\
\text { Pankreozy- } \\
\text { min/kg }\end{array}$} \\
\hline & $\overline{\mathbf{x}}$ & $S \bar{x}$ & $\overline{\mathbf{x}}$ & $S \bar{x}$ & $\overline{\mathbf{x}}$ & $S \bar{x}$ \\
\hline $0-10$ & 32 & 4,55 & 31 & 3,50 & 19 & 2,09 \\
\hline $10-20$ & 38 & 6,39 & 30 & 3,44 & 22 & 4,84 \\
\hline $20-30$ & 32 & 6,72 & 24 & 3,11 & 17 & 4,13 \\
\hline $30-40$ & 22 & 4,34 & 22 & 3,02 & 18 & 2,43 \\
\hline $40-50$ & 23 & 4,39 & 20 & 1,96 & 15 & 3,05 \\
\hline $50-60$ & 22 & 4,13 & 20 & 1.97 & 15 & 3,11 \\
\hline
\end{tabular}

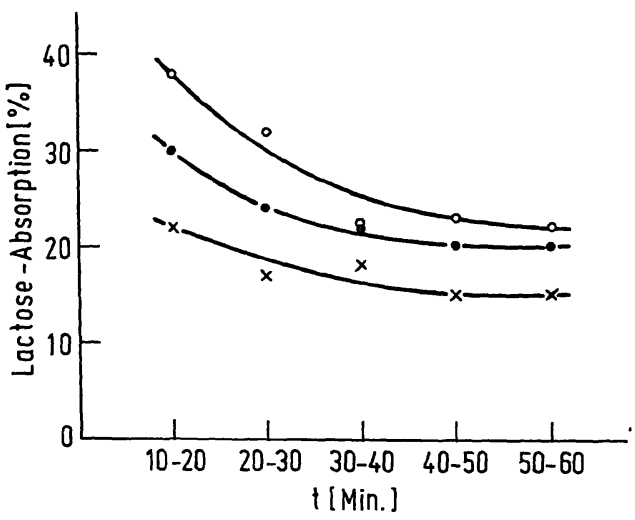

Abb. 1

Lactoseabsorption bei unbehandelten Tieren o, nach Sekretingabe * und Pankreozymingabe $\mathrm{x}$ in Prozent vom Ausgangswert.

unbehandelten Tieren. Die Unterschiede zwischen unbehandelten Tieren und Tieren nach Sekretingabe sind zu keiner Zeit statistisch signifikant, während die Lactosedigestion in den ersten drei Fraktionen nach Pankreozymingabe signifikant geringer ist als bei unbehandelten Tieren.

Das Ausmaß der Wasserverschiebung ist aus Tabelle 2 und Abbildung 2 zu ersehen. Der höchste Anstieg der Kollidonkonzentrationen findet sich bei unbehandelten Tieren, während nach Sekretin- und Pankreozymingabe der Konzentrationseffekt wesentlich geringer ist. Eine statistische Signifikanz besteht jedoch nur zwischen Kontrolltieren und Tieren nach Pankreozymingabe in der ersten Fraktion (0-10 Min. nach Perfusionsbeginn).
Tab. 2

Kollidonkonzentrationen in Prozent vom Ausgangswer

\begin{tabular}{|c|c|c|c|c|c|c|}
\hline \multirow{2}{*}{$\begin{array}{l}\text { Perfusionsdauer } \\
\text { (Min.) }\end{array}$} & \multicolumn{2}{|c|}{ Kontrolltiere } & \multicolumn{2}{|c|}{$\underset{\text { Sekretin/kg }}{\operatorname{nach} 6 \text { E. }}$} & \multicolumn{2}{|c|}{$\begin{array}{c}\text { nach } 6 \text { E. } \\
\text { Pankreozy } \\
\text { min } / \mathrm{kg}\end{array}$} \\
\hline & $\overline{\mathbf{x}}$ & $S \bar{x}$ & $\overline{\mathbf{x}}$ & $\mathbf{S} \overline{\mathbf{x}}$ & & $\mathbf{s} \bar{x}$ \\
\hline $0-10$ & 137 & $\cdot 9,53$ & 127 & 8,99 & 117 & 3,71 \\
\hline $10-20$ & 144 & 12,77 & 127 & 7,87 & 122 & 6,30 \\
\hline $20-30$ & 137 & 10,83 & 118 & 2,93 & 117 & 5,89 \\
\hline $30-40$ & 125 & 6,02 & 117 & 2,54 & 118 & 3,18 \\
\hline $40-50$ & 120 & 5,19 & 117 & 3,16 & 113 & 4,13 \\
\hline $50-60$ & 118 & 4,57 & 118 & 2,62 & 115 & 3,72 \\
\hline
\end{tabular}

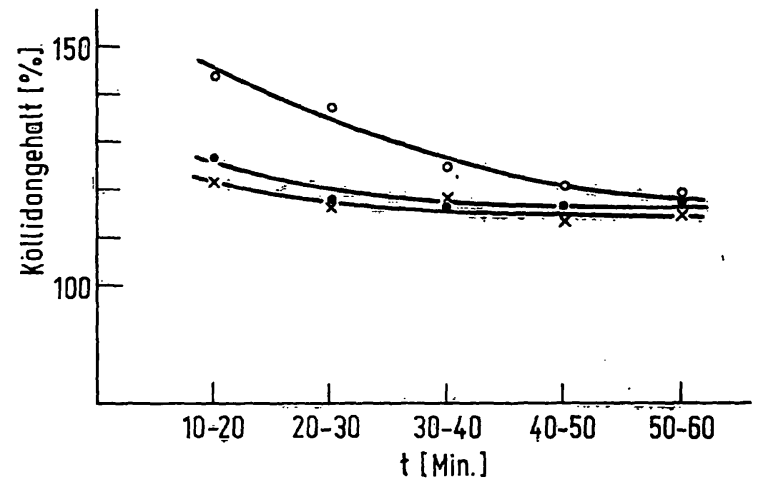

Abb. 2

Kollidongehalt der Perfusionslösung bei unbehandelten Tieren o, nach Sekretingabe - und Pankreozymingabe $x$ in Prozent vom Ausgangswert

Tab. 3

Konzentration $(\dot{\bar{x}})$ der freien Galaktose $(\mathrm{mg} / 100 \mathrm{ml})$ in der Perfusionslösung nach Dünndarmpassage

\begin{tabular}{|c|c|c|c|c|c|c|}
\hline $\begin{array}{c}\text { Perfusionsdauer } \\
\text { (Min.) }\end{array}$ & $0-10$ & $10-20$ & $20-30$ & $30-40$ & $40-50$ & $50-60$ \\
\hline Kontrolltiere & 1,1 & 1,0 & 1,0 & 1,2 & 1,2 & 1,4 \\
\hline $\begin{array}{l}\text { Nach Sekretion } \\
\text { i. v. }\end{array}$ & 1,3 & 1,0 & 1,2 & 1,2 & 1,3 & 1,3 \\
\hline $\begin{array}{l}\text { Nach } \\
\text { Pankreozymin } \\
\text { i. v. }\end{array}$ & 0,8 & 1,0 & 1,3 & 1,2 & 1,3 & 1,1 \\
\hline
\end{tabular}

Die Mengen der nach der Darmpasasge in der Perfusionslösung enthaltenen, noch nicht resorbierten freien Galaktose liegen sowohl bei den Kontrolltieren als auch nach Sekretin- und Pankreozymingabe zwischen 0,8 und $1,4 \mathrm{mg} / 100 \mathrm{ml}$ (Tab. 3). Ein statistischer Vergleich wurde nicht angestellt, da bei diesen niedrigen Konzentrationen die Schwankung im Streubereich der Methode liegt.

\section{Diskussion}

Sowohl bei unbehandelten Kontrolltieren, als nach Sekretin- und Pankreozymingabe, nahm die Lactosedigestion mit zunehmender Perfusionsdauer $a b$ (Abb. 1). Im Zeitraum 10-20 Min. nach Perfusionsbeginn wurden von Kontrolltieren $38 \%$ der angebotenen Lactose gespalten, während am Ende des Versuches (50-60 Min. nach Perfusionsbeginn) nur noch $22 \%$ gespalten wurden. Ähnlich lagen die Verhältnisse nach Sekretin (30\% und $20 \%$ ) und Pankreozymin (22\% und 15\%) in der erwähnten Zeiträumen. Dieser Effekt beruht möglicherweise auf einem Erschöpfen der Laktaseaktivität der Mukosazellen. 
Im Zeitraum 0-10 Min. nach Perfusionsbeginn war in allen 3 Kollektiven die Lactoseverwertung scheinbar geringer als im folgenden Zeitabschnitt 10-20 Min. (Tab. 1). Dieser Effekt ist jedoch Folge der angewandten Technik, da zu Beginn der Perfusion der Darm und der abführende Schlauch schnell $(5 \mathrm{~m} / /$ Min.) mit der Perfusionslösung aufgefüllt wurden und in dieser kurzen Zeit praktisch keine Lactose gespalten werden konnte, so daß zunächst unveränderte Perfusionslösung mit der ersten Probe aufgefangen wurde. Das gleiche trifft für die Wasserabsorption, gemessen an der Kollidonkonzentration, zu. Aus diesem Grunde wurde der Zeitraum 0-10 Min. nach Perfusionsbeginn in den Abbildungen 1 und 2 nicht berücksichtigt.

Nach i. v.-Gabe von 6,0 E. Sekretin/kg Körpergewicht fanden wir eine statistisch nicht signifikante Verminderung der Lactosedigestion, während nach $6,0 \mathrm{E}$. Pankreozymin $/ \mathrm{kg}$ Körpergewicht in den ersten drei Fraktionen eine signifikante Verminderung der Lactoseverwertung festzustellen war. Die in den drei letzten Proben gefundenen Unterschiede waren nicht mehr signifikant.

Da vor der Lactosebestimmung ein getrennter Nachweis der freien Galaktose erfolgte, ist die Möglichkeit ausgeschlossen, daß durch Miterfassen der Galaktose zu hohe Lactosewerte erhalten wurden.

Die Hemmung der Lactosehydrolyse durch Pankreozymin kann verschiedene Ursachen haben. Einmal kann primär der Lactosetransport vom Darmlumen in die Mukosazelle gestört sein, zum anderen wäre auch eine Hemmung der Lactaseaktivität im Bürstensaum der Mukosazelle möglich mit sekundärer Inhibierung des Lactosetransportes. Die Hemmung des Transportsystems wie auch die Hemmung der Lactaseaktivität könnten direkte Folge des Pankreozymineinflusses sein. Ferner muß ein indirekter Wirkungsmechanismus über das endokrine Pankreas nach Pankreozyminreiz diskutiert werden. Es ist bekannt, daß Sekretin und Pan- kreozymin eine Insulinfreisetzung bewirken $(4,5)$. Nach Beyretss, Müller und Strack (6) hemmt Insulin die Galaktoseabsorption beim Kaninchen. In unseren Versuchen zeigte sich jedoch nach Sekretin- und Pankreozymingabe keine Hemmung der Galaktoseabsorption. Die Galaktosekonzentrationen im Perfusat lagen sowohl bei den Kontrolltieren als auch nach Sekretin- und Pankreozymingabe im gleichen niedrigen Bereich um $1 \mathrm{mg} /$ $100 \mathrm{~m} /$. Bei einer Hemmung der Galaktoseabsorption müBte sich jedoch ein erhöhter Galaktosespiegel finden. Die fehlende Beeinflussung der Galaktoseabsorption durch Insulin könnte jedoch dadurch erklärt werden, $\mathrm{da} B$ in der von uns verwendeten Perfusionslösung die Lactosekonzentration mit $100 \mathrm{mg} / 100 \mathrm{~m} l$ relativ gering war gegenüber der von BEYREIss ( 6 ) benutzten Lösung. Es ist möglich, daß der durch Insulin hervorgerufene Hemmungsmechanismus erst bei höheren Galaktosekonzentrationen sichtbar wird. Bisher ist nicht bekannt, ob die Höhe des Insulinspiegels im Serum auch die Lactosedigestion beeinflußt.

Das Ausmaß der Wasserverschiebung ist bei Kontrolltieren ausgeprägter als nach Sekretin- und Pankreozymingabe (Abb. 2). Die Kollidonkonzentrationen liegen bei Normaltieren deutlich höher, es erfolgte ein Wasseraustritt aus dem Darmlumen. Ein signifikanter Unterschied besteht jedoch nur zwischen der 1. Fraktion (0-10 Min.) nach Pankreozymingabe und der bei Normaltieren, die übrigen Werte unterscheiden sich nicht signifikant voneinander.

Eine Verdünnung des Darminhaltes durch Pankreassekret als Folge einer Steigerung der hydrokinetischen Funktion nach Pankreasstimulation scheidet aus, da der Darm am Beginn der Perfusionsstrecke nach proximal abgebunden war und aus dem oberen Darmabschnitt keine Flüssigkeit in das perfundierte Stück gelangen konnte. Es handelt sich um eine Beeinflussung des Darmes entweder im Sinne einer verminderten Wasserexsorption oder einer gesteigerten Insorption.

\section{Literatur}

1. DAkrQvist, A. und B. Borgström, Biochem. J. 81, 411 (1961). 2. Dafrquist, A. und D. L. Thomson, Acta physiol. scand. 61, 20 (1964). - 3. Schubert, R. und H. Werner, Z. exper. Med. 131, 90 (1959). - 4. Pfeiffer, E. F., M. Telib, J. Ammon, F.
Mrlani und H. Ditschuneit, Dtsch. med. Wschr. 90, 1201 (1965). - 5. Unger, R. H., H. Ketterer, J. Dupré und A. M. Eisentraut, J. Clin. Invest., 4, 630 (1967). - 6. Beyrerss, K., F. Müller und E. StraCk, Z. exper. Med. 138, 277 (1964).
Priv.-Doz. Dr. K. Rommel Dr. R. Böhmer $7900 \mathrm{Ulm}$ Steinhövelstr. 9 